\title{
Facile Synthesis of Amine-Terminated Aromatic Polyamide Dendrimer via Divergent Method
}

Isao Washio, Yuji Shibasaki, and Mitsuru Ueda*

Department of Organic and Polymeric Materials, Graduate School of Science and Engineering, Tokyo Institute of Technology, 2-12-1, O-okayama, Meguro-ku, Tokyo 152-8552, Japan

Supporting Information 
Figure 1S. ${ }^{1} \mathrm{H}$ NMR spectrum of 3,5-bis(trifluoroacetamido)benzoic acid

Figure 2S. ${ }^{13} \mathrm{C}$ NMR spectrum of 3,5-bis(trifluoroacetamido)benzoic acid

Figure 3S. ${ }^{1} \mathrm{H}$ NMR spectrum of 3,5-bis(trifluoroacetamido)benzoyl cloride (1)

Figure 4S. ${ }^{1} \mathrm{H}$ NMR spectrum of G1 dendrimer $\left(\mathbf{N H}_{2}-\mathbf{G 1 D}\right)$

Figure 5S. ${ }^{13} \mathrm{C}$ NMR spectrum of $\mathrm{G} 1$ dendrimer $\left(\mathbf{N H}_{2}-\mathbf{G 1 D}\right)$

Figure 6S. ${ }^{1} \mathrm{H}$ NMR spectrum of G2 dendrimer $\left(\mathbf{N H}_{\mathbf{2}}-\mathbf{G} \mathbf{- 2 D}\right)$

Figure 7S. ${ }^{13} \mathrm{C}$ NMR spectrum of $\mathrm{G} 2$ dendrimer $\left(\mathbf{N H}_{2}\right.$-G2D)

Figure 8S. MALDI-TOF MS spectrum of G2 dendrimer ( $\left.\mathbf{N H}_{\mathbf{2}} \mathbf{- G 2 D}\right)$

Figure 9S. ${ }^{1} \mathrm{H}$ NMR spectrum of G3 dendrimer $\left(\mathbf{N H}_{2}\right.$-G3D)

Figure 10S. ${ }^{13} \mathrm{C}$ NMR spectrum of $\mathrm{G} 3$ dendrimer $\left(\mathbf{N H}_{\mathbf{2}}-\mathbf{G} 3 \mathrm{D}\right)$

Figure 11S. MALDI-TOF MS spectrum of protected-G3 dendrimer $\left(\mathbf{C F}_{\mathbf{3}} \mathbf{- G 3 D}\right)$

Figure 12S. ${ }^{1} \mathrm{H}$ NMR spectrum of $\mathrm{G} 4$ dendrimer $\left(\mathbf{N H}_{\mathbf{2}}-\mathbf{G} 4 \mathbf{D}\right)$

Figure $13 \mathrm{~S} .{ }^{13} \mathrm{C}$ NMR spectrum of G4 dendrimer $\left(\mathbf{N H}_{\mathbf{2}}-\mathbf{G} 4 \mathrm{D}\right)$

Figure 14S. MALDI-TOF MS spectrum of protected-G4 dendrimer $\left(\mathbf{C F}_{\mathbf{3}} \mathbf{- G 4 D}\right)$

Figure 15S. GPC traces of amine-terminated dendrimers $\left(\mathbf{N H}_{\mathbf{2}}-\mathbf{G n D}\right)$ 
Measurement. Infrared spectra were recorded on a Horiba FT-720 spectrophotometer. ${ }^{1} \mathrm{H}$ and ${ }^{13} \mathrm{C}$ NMR spectra were obtained on a BRUKER DPX-300 spectrometer at 300 and $75 \mathrm{MHz}$, respectively. Thermal analysis was performed on a Seiko EXSTAR 6000 TG/DTA 6300 thermal analyzer at a heating rate of $10{ }^{\circ} \mathrm{C} / \mathrm{min}$ for thermogravimetry (TG) and differential thermal analysis (DTA), and a Seiko EXSTAR 6000 DSC 6200 at a heating rate of $10{ }^{\circ} \mathrm{C} / \mathrm{min}$ for differential scanning calorimetry (DSC) under nitrogen. Matrix-assisted laser desorption ionization with time of flight (MALDI-TOF) mass spectrum of $\mathbf{N H}_{\mathbf{2}}-\mathbf{G 2 D}$ was obtained on a Kratos Kompact MALDI instrument operated in linear detection mode to generate positive ion spectra using dithranol as a matrix, $N, N$-dimethylformamide (DMF) as a solvent, and sodium trifluoroacetate as an additive agent. For $\mathbf{C F}_{\mathbf{3}} \mathbf{- G 3 D}$ and $\mathbf{C F}_{\mathbf{3}} \mathbf{- G 4 D}$ analyses, BRUKER DALTONICS autoflex instrument was used in reflector detection mode to generate positive ion spectra using dithranol as a matrix, tetrahydrofuran (THF) as a solvent, and sodium trifluoroacetate as an additive agent. Number- and weight-average molecular weights $\left(M_{\mathrm{n}}\right.$ and $\left.M_{\mathrm{w}}\right)$ were determined by a gel permeation chromatograph (GPC) on a Jasco co-2065 GPC system equipped with polystyrene gel columns (TSK GELs, $\mathrm{GMH}_{\mathrm{HR}}-\mathrm{M}$ and $\mathrm{GMH}_{\mathrm{HR}}-\mathrm{L}$ ) eluted with DMF containing $0.01 \mathrm{M}$ of lithium bromide at a flow rate of $1.0 \mathrm{ml} / \mathrm{min}$ and calibrated by standard polystyrene samples. 
Materials. $N$-Methyl-2-pyrrolidinone (NMP) was distilled under reduced pressure over calcium hydride and then stored under nitrogen. THF was dried over sodium and distilled before use under nitrogen. $p$-Phenylenediamine was purchased from Kanto Kagaku Co. and used as received. The other reagents and solvents were obtained commercially and used as received.

\section{Synthesis of 3,5-bis(trifluoroacetamido)benzoic acid}

To a solution of 3,5-diaminobenzoic acid (12.2 $\mathrm{g}, 80.0 \mathrm{mmol})$ in THF (100 ml) was added trifluoroacetic anhydride $(54.6 \mathrm{~g}, 260 \mathrm{mmol})$ at $0{ }^{\circ} \mathrm{C}$ under nitrogen, and stirred at the temperature for $10 \mathrm{~min}$, subsequently $25^{\circ} \mathrm{C}$ for $3 \mathrm{~h}$. Then, water $(100 \mathrm{ml})$ was added and the resultant solution was stirred for $6 \mathrm{~h}$ at the temperature. The reaction mixture was extracted with diethyl ether. The organic layer was washed with water several times, dried over $\mathrm{MgSO}_{4}$, and filtered. The filtrate was evaporated, and the residue was recrystallized from acetonitrile and dried under reduced pressure at $120{ }^{\circ} \mathrm{C}$ to give pale brown plates ( $94 \%$ yield).

Mp (DTA): $262{ }^{\circ} \mathrm{C} . \quad$ IR (KBr): 713, 1168 (C-F), 1570, 1616 (Ar-H), 1716 (C=O), 2500 3300 (O-H), and $3313 \mathrm{~cm}^{-1}(\mathrm{~N}-\mathrm{H}) .{ }^{1} \mathrm{H}$ NMR (DMSO, $\left.40{ }^{\circ} \mathrm{C}\right): \delta=8.12(\mathrm{~d}, 2 \mathrm{H})$, 
$8.39(\mathrm{t}, 1 \mathrm{H}), 11.46(\mathrm{~s}, 2 \mathrm{H}) .{ }^{13} \mathrm{C} \mathrm{NMR}\left(\mathrm{CDCl}_{3}\right): \delta=115.8(\mathrm{q}, \mathrm{J}=286.7 \mathrm{~Hz}), 117.3,118.9$,

$132.4,137.4,155.0(\mathrm{q}, \mathrm{J}=37.0 \mathrm{~Hz})$, and $166.5 \mathrm{ppm}$.

\section{Synthesis of 3,5-bis(trifluoroacetamido)benzoyl chloride (1)}

3,5-Bis(trifluoroacetamido)benzoic acid (10.0 g, $29.1 \mathrm{mmol})$ was refluxed in $100 \mathrm{ml}$ of thionyl chloride for $6 \mathrm{~h}$ under nitrogen. The thionyl chloride was distilled off, and the residue was dissolved in tetrachloroethane at $100{ }^{\circ} \mathrm{C}$ and subsequently cooled to $20{ }^{\circ} \mathrm{C}$ to be recrystallized. The precipitate was filtrated and washed with hexane, followed by recrystallization again from dehydrated chloroform. The product was filtered and dried at $60{ }^{\circ} \mathrm{C}$ under reduced pressure to give slightly yellow needles (55\% yield).

Mp: $145-147{ }^{\circ} \mathrm{C} . \quad$ IR (KBr): 702, 1188 (C-F), 1022 (C-Cl), 1562, 1616 (Ar-H), 1716

$(\mathrm{C}=\mathrm{O})$, and $3286 \mathrm{~cm}^{-1}(\mathrm{~N}-\mathrm{H}) .{ }^{1} \mathrm{H} \mathrm{NMR}\left(\mathrm{CDCl}_{3}, 25^{\circ} \mathrm{C}\right): \delta=8.19(\mathrm{~d}, 2 \mathrm{H}), 8.30(\mathrm{~s}, 2 \mathrm{H})$, $8.45(\mathrm{t}, 1 \mathrm{H})$. Anal. Calcd for $\mathrm{C}_{11} \mathrm{H}_{5} \mathrm{ClF}_{6} \mathrm{~N}_{2} \mathrm{O}_{3}: \mathrm{C}, 36.43 \% ; \mathrm{H}, 1.39 \% ; \mathrm{N}, 7.73 \%$. Found: C, 36.31\%; H, 1.64\%; N, 7.51\%

\section{Synthesis of G1 dendrimer ( $\left.\mathrm{NH}_{2}-\mathrm{G1D}\right)$}

To a solution of $p$-phenylenediamine $(0.108 \mathrm{~g}, 1.00 \mathrm{mmol})$ in NMP $(2 \mathrm{ml})$ was added $\mathrm{AB}_{2}$-building block $1(0.798 \mathrm{~g}, 2.20 \mathrm{mmol})$ at $0{ }^{\circ} \mathrm{C}$ under nitrogen, and stirred at the 
temperature for $5 \mathrm{~min}$, followed by $25^{\circ} \mathrm{C}$ for $1 \mathrm{~h}$. Then, water $(10 \mathrm{mg})$ was added, after which stirring was continued for $1 \mathrm{~h}$ at $50{ }^{\circ} \mathrm{C}$. The resultant solution was treated with hydrazine monohydrate $(0.600 \mathrm{~g}, 12.0 \mathrm{mmol})$ for another $1 \mathrm{~h}$ at the temperature. The reaction mixture was poured into $2 \mathrm{wt} \%$ of $\mathrm{NaHCO}_{3 a q}$, subsequently the precipitate was filtered and dried at $120{ }^{\circ} \mathrm{C}$ under reduced pressure to give white solid (>99\% yield). Mp (DTA): $341{ }^{\circ} \mathrm{C} . \quad$ IR (KBr): 1519, 1554, $1620(\mathrm{Ar}-\mathrm{H}), 1639(\mathrm{C}=\mathrm{O})$, and 1597, 3409 $\mathrm{cm}^{-1}(\mathrm{~N}-\mathrm{H}) . \quad{ }^{1} \mathrm{H}$ NMR (DMSO, $\left.40{ }^{\circ} \mathrm{C}\right): \delta=4.83(\mathrm{~s}, 8 \mathrm{H}), 6.00(\mathrm{t}, 2 \mathrm{H}), 6.31(\mathrm{~d}, 4 \mathrm{H}), 7.64$ (s, 4H), 9.76 (s, 2H). ${ }^{13} \mathrm{C}$ NMR (DMSO $40{ }^{\circ} \mathrm{C}$ ): $\delta=102.2,102.3,120.1,134.9,136.8$, 148.9, and 166.8 ppm. Anal. Calcd for $\mathrm{C}_{20} \mathrm{H}_{20} \mathrm{~N}_{6} \mathrm{O}_{2}:$ C, 63.8\%; H, 5.36\%; N, 22.33\%. Found: C, 64.12\%; H, 5.64\%; N, 22.37\%.

\section{Synthesis of $\mathbf{G} 2$ dendrimer $\left(\mathrm{NH}_{2}-\mathrm{G} 2 \mathrm{D}\right)$}

To a solution of $\mathbf{N H}_{2}$-G1D $(0.0941 \mathrm{~g}, 0.250 \mathrm{mmol})$ in NMP (1 ml) was added $\mathrm{AB}_{2}$-building block $1(0.399 \mathrm{~g}, 1.1 \mathrm{mmol})$ at $0{ }^{\circ} \mathrm{C}$ under nitrogen, and stirred at the temperature for $5 \mathrm{~min}$, followed by $25^{\circ} \mathrm{C}$ for $1 \mathrm{~h}$. Then, water $(5 \mathrm{mg})$ was added, after which stirring was continued for $1 \mathrm{~h}$ at $50{ }^{\circ} \mathrm{C}$. The resultant solution was treated with hydrazine monohydrate $(0.300 \mathrm{~g}, 12.0 \mathrm{mmol})$ for another $1 \mathrm{~h}$ at the temperature. The 
reaction mixture was poured into $2 \mathrm{wt} \%$ of $\mathrm{NaHCO}_{3 a q}$, subsequently the precipitate was filtered and dried at $120{ }^{\circ} \mathrm{C}$ under reduced pressure to give white solid (94\% yield). Mp (DTA): $371{ }^{\circ} \mathrm{C} . \quad$ IR (KBr): 1512, $1554(\mathrm{Ar}-\mathrm{H}), 1639(\mathrm{C}=\mathrm{O})$, and 1601, $3325 \mathrm{~cm}^{-1}$ (N-H). ${ }^{1} \mathrm{H}$ NMR (DMSO, $\left.40{ }^{\circ} \mathrm{C}\right): \delta=4.85(\mathrm{~s}, 16 \mathrm{H}), 6.03(\mathrm{t}, 4 \mathrm{H}), 6.37(\mathrm{~d}, 8 \mathrm{H}), 7.73(\mathrm{~s}$, 4H), $7.91(\mathrm{~d}, 4 \mathrm{H}), 8.35(\mathrm{t}, 2 \mathrm{H}), 10.01(\mathrm{~s}, 4 \mathrm{H}) 10.20(\mathrm{~s}, 2 \mathrm{H}) .{ }^{13} \mathrm{C}$ NMR (DMSO, $\left.40{ }^{\circ} \mathrm{C}\right): \delta$ $=102.5,114.9,115.4,120.4,134.9,136.0,136.5,139.5,148.9,165.8$ and $167.1 \mathrm{ppm}$. Anal. Calcd for $\mathrm{C}_{48} \mathrm{H}_{44} \mathrm{~N}_{14} \mathrm{O}_{6} 1.36 \mathrm{H}_{2} \mathrm{O}$ : C, 61.49\%; H, 5.02\%; N, 20.92\%. Found: $\mathrm{C}$, 61.67\%; H, 5.20\%; N, 21.02\%. Calcd.: $[\mathrm{M}]^{+} \mathrm{m} / \mathrm{z}=912.4$. Found: MALDI-TOF-MS: $[\mathrm{M}+\mathrm{Na}]^{+}=936.4$ and $[\mathrm{M}+\mathrm{K}]^{+}=952.5$.

\section{Synthesis of G3 dendrimer ( $\left.\mathrm{NH}_{2}-\mathrm{G} 3 D\right)$}

To a solution of $\mathbf{N H}_{\mathbf{2}}$-G2D $(0.114 \mathrm{~g}, 0.125 \mathrm{mmol})$ in NMP $(1.33 \mathrm{ml})$ was added $\mathrm{AB}_{2}$-building block $1(0.399 \mathrm{~g}, 1.1 \mathrm{mmol})$ at $0{ }^{\circ} \mathrm{C}$ under nitrogen, and stirred at the temperature for $5 \mathrm{~min}$, followed by $25^{\circ} \mathrm{C}$ for $1 \mathrm{~h}$. Then, water $(5 \mathrm{mg})$ was added, after which stirring was continued for $1 \mathrm{~h}$ at $50{ }^{\circ} \mathrm{C}$. The resultant solution was treated with hydrazine monohydrate $(0.300 \mathrm{~g}, 12.0 \mathrm{mmol})$ for another $3 \mathrm{~h}$ at the temperature. The reaction mixture was poured into $2 \mathrm{wt} \%$ of $\mathrm{NaHCO}_{3 a q}$, subsequently the precipitate was filtered and dried at $120{ }^{\circ} \mathrm{C}$ under reduced pressure to give white solid (95\% yield). The 
intermediate, $\mathbf{C F}_{\mathbf{3}} \mathbf{- G 3 D}$, was isolated to obtain the MALDI-TOF MS spectrum. The coupling reaction of $\mathbf{N H}_{2}-\mathbf{G} 2 \mathbf{D}$ and $\mathbf{1}$ was carried out to yield $\mathbf{C F}_{\mathbf{3}}-\mathbf{G} 3 \mathbf{D}$ using the same protocol as described above. The reaction solution was poured into $1 \mathrm{wt} \%$ of $\mathrm{NaHCO}_{3 \mathrm{aq}}$, and then the precipitate was filtered and dried at $80{ }^{\circ} \mathrm{C}$ under reduced pressure. The resulting product was analyzed by MALDI-TOF MS spectroscopy.

$\mathrm{T}_{\mathrm{g}}$ (glass-transition temperature): $276{ }^{\circ} \mathrm{C} . \quad \mathrm{IR}(\mathrm{KBr}): 1543(\mathrm{Ar}-\mathrm{H}), 1639(\mathrm{C}=\mathrm{O})$, and 1601, $3332 \mathrm{~cm}^{-1}(\mathrm{~N}-\mathrm{H}) . \quad{ }^{1} \mathrm{H}$ NMR (DMSO, $\left.40{ }^{\circ} \mathrm{C}\right): \delta=4.74(\mathrm{~s}, 32 \mathrm{H}), 6.06(\mathrm{t}, 8 \mathrm{H}), 6.42$ (d, 16H), $7.76(\mathrm{~s}, 4 \mathrm{H}), 7.98(\mathrm{~d}, 8 \mathrm{H}), 8.01(\mathrm{~d}, 4 \mathrm{H}), 8.35(\mathrm{t}, 4 \mathrm{H}), 8.46(\mathrm{t}, 2 \mathrm{H}), 9.89(\mathrm{~s}, 8 \mathrm{H})$, $10.16(\mathrm{~s}, 2 \mathrm{H}), 10.33(\mathrm{~s}, 4 \mathrm{H}) .{ }^{13} \mathrm{C}$ NMR (DMSO, $\left.40{ }^{\circ} \mathrm{C}\right): \delta=102.6,102.7,115.1,115.3$, $115.7,120.6,134.9,135.7,136.2,136.5,139.3,139.5,148.8,165.6,166.1$ and $167.0 \mathrm{ppm}$. Anal. Calcd for $\mathrm{C}_{104} \mathrm{H}_{92} \mathrm{~N}_{30} \mathrm{O}_{14} 3.77 \mathrm{H}_{2} \mathrm{O}: \mathrm{C}, 60.81 \%$; H, 4.88\%; N, 20.46\%. Found: C, 60.51\%; H, 4.58\%; N, 20.51\%. Calcd. for $\mathbf{C F}_{3}-\mathbf{G 3 D}:[\mathrm{M}]^{+} \mathrm{m} / \mathrm{z}=3520.5$. Found: MALDI-TOF-MS: $[\mathrm{M}+\mathrm{Na}]^{+}=3543.5$ and $[\mathrm{M}+2 \mathrm{Na}-\mathrm{H}]^{+}=3565.5$.

\section{Synthesis of G4 dendrimer ( $\left.\mathrm{NH}_{2}-\mathrm{G} 4 \mathrm{D}\right)$}

To a solution of $\mathbf{N H}_{\mathbf{2}}$-G3D $(0.124 \mathrm{~g}, 0.0625 \mathrm{mmol})$ in NMP $(1.33 \mathrm{ml})$ was added $\mathrm{AB}_{2}$-building block $1(0.399 \mathrm{~g}, 1.1 \mathrm{mmol})$ at $0{ }^{\circ} \mathrm{C}$ under nitrogen, and stirred at the 
temperature for $5 \mathrm{~min}$, followed by $25^{\circ} \mathrm{C}$ for $1 \mathrm{~h}$. Then, water $(5 \mathrm{mg})$ was added, after which stirring was continued for $1 \mathrm{~h}$ at $50{ }^{\circ} \mathrm{C}$. The resultant solution was treated with hydrazine monohydrate $(0.300 \mathrm{~g}, 12.0 \mathrm{mmol})$ for another $3 \mathrm{~h}$ at the temperature. The reaction mixture was poured into $2 \mathrm{wt} \%$ of $\mathrm{NaHCO}_{3 a q}$, subsequently the precipitate was filtered and dried at $120{ }^{\circ} \mathrm{C}$ under reduced pressure to give white solid (95\% yield). The intermediate, $\mathbf{C F}_{\mathbf{3}} \mathbf{- G 4 D}$, was isolated to obtain the MALDI-TOF MS spectrum. The coupling reaction of $\mathbf{N H}_{2}-\mathbf{G} 3 \mathbf{D}$ and $\mathbf{1}$ was carried out to yield $\mathbf{C F}_{\mathbf{3}}-\mathbf{G} \mathbf{-} \mathbf{D}$ using the same protocol as described above. The reaction solution was poured into $1 \mathrm{wt} \%$ of $\mathrm{NaHCO}_{3 \mathrm{aq}}$, and then the precipitate was filtered and dried at $80{ }^{\circ} \mathrm{C}$ under reduced pressure. The resulting product was analyzed by MALDI-TOF MS spectroscopy.

$\mathrm{T}_{\mathrm{g}}: 300{ }^{\circ} \mathrm{C} . \quad \mathrm{IR}(\mathrm{KBr}): 1543(\mathrm{Ar}-\mathrm{H}), 1639(\mathrm{C}=\mathrm{O})$, and $1601,3321 \mathrm{~cm}^{-1}(\mathrm{~N}-\mathrm{H}) .{ }^{1} \mathrm{H}$ NMR (DMSO, $\left.40{ }^{\circ} \mathrm{C}\right): \delta=4.75(\mathrm{~s}, 64 \mathrm{H}), 6.04(\mathrm{t}, 16 \mathrm{H}), 6.41(\mathrm{~d}, 32 \mathrm{H}), 7.75(\mathrm{~s}, 4 \mathrm{H}), 7.98(\mathrm{~d}$, $16 \mathrm{H}), 8.03(\mathrm{~d}, 4 \mathrm{H}), 8.06(\mathrm{~d}, 8 \mathrm{H}), 8.37(\mathrm{t}, 8 \mathrm{H}), 8.46-8.52(\mathrm{~m}, 6 \mathrm{H}), 9.90(\mathrm{~s}, 16 \mathrm{H}), 10.17(\mathrm{~s}$, 2H), 10.35 (s, 8H), 10.44 (s, 4H). ${ }^{13} \mathrm{C}$ NMR (DMSO, $\left.40{ }^{\circ} \mathrm{C}\right): \delta=102.6,102.7,115.1$, $115.4,115.7,120.6,134.9,135.7,136.0,136.2,136.4,139.3,139.5,148.8,165.5,166.0$, 166.1 and 167.0 ppm. Anal. Calcd for $\mathrm{C}_{216} \mathrm{H}_{188} \mathrm{~N}_{62} \mathrm{O}_{30} 8.88 \mathrm{H}_{2} \mathrm{O}: \mathrm{C}, 60.44 \% ; \mathrm{H}, 4.83 \%$; N, 20.23\%. Found: C, 60.45\%; H, 4.84\%; N, 20.24\%. Calcd. for $\mathbf{C F}_{3}-\mathbf{G} 4 \mathrm{D}:[\mathrm{M}]^{+} \mathrm{m} / \mathrm{z}$ $=7204.5$. Found: MALDI-TOF-MS: $[\mathrm{M}+\mathrm{Na}]^{+}=7228.3$. 
- 10 - 

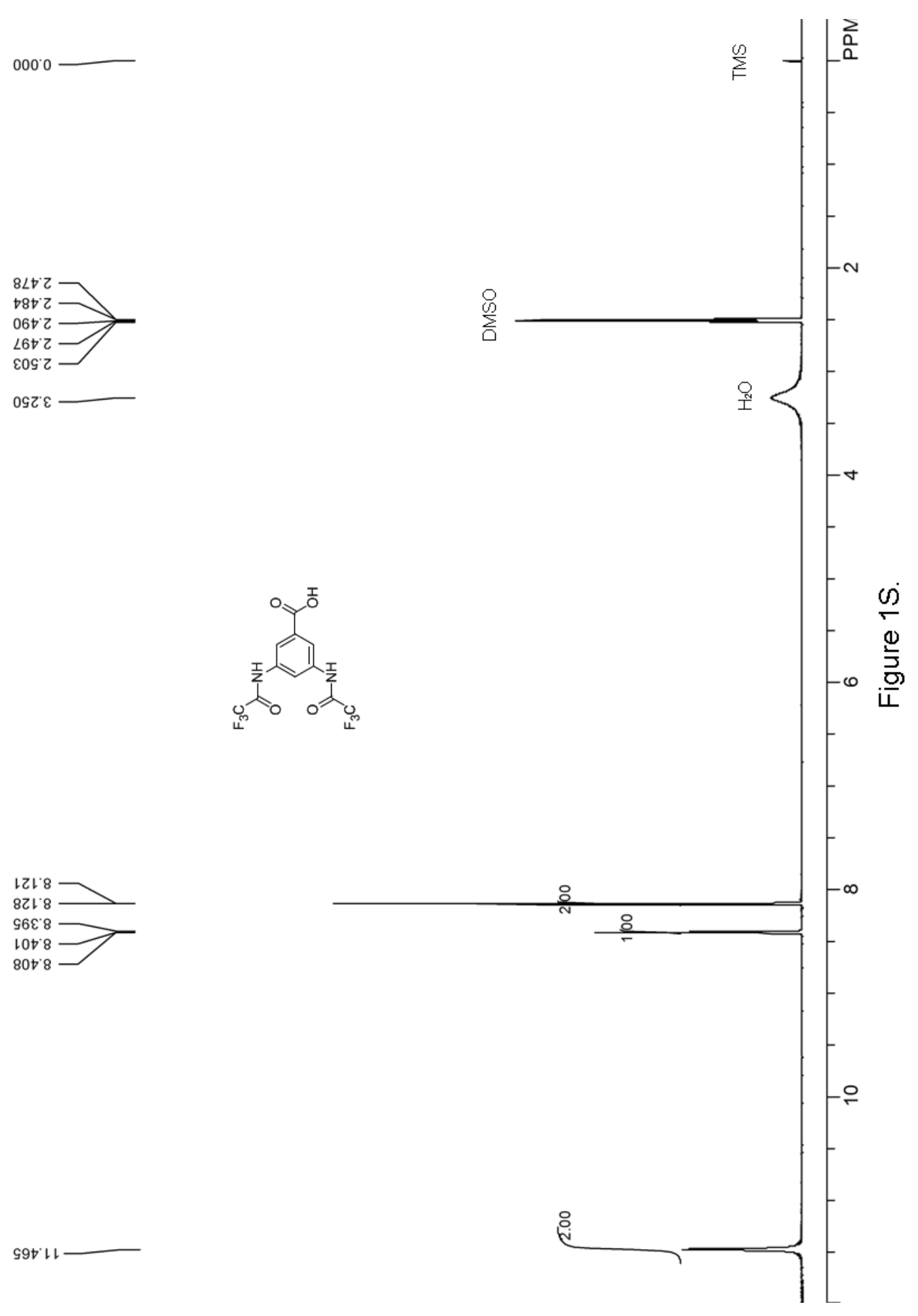

s9t1 

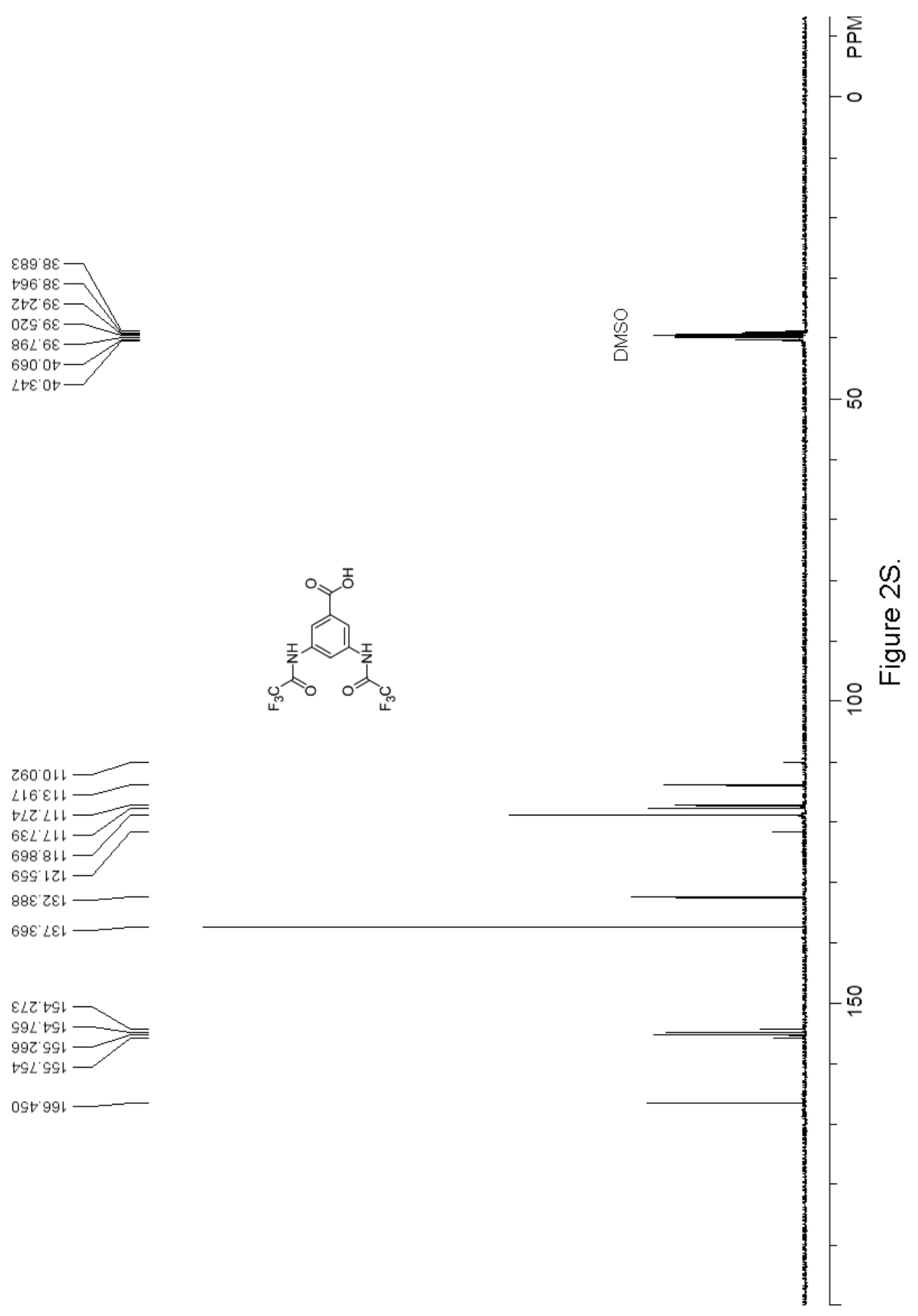


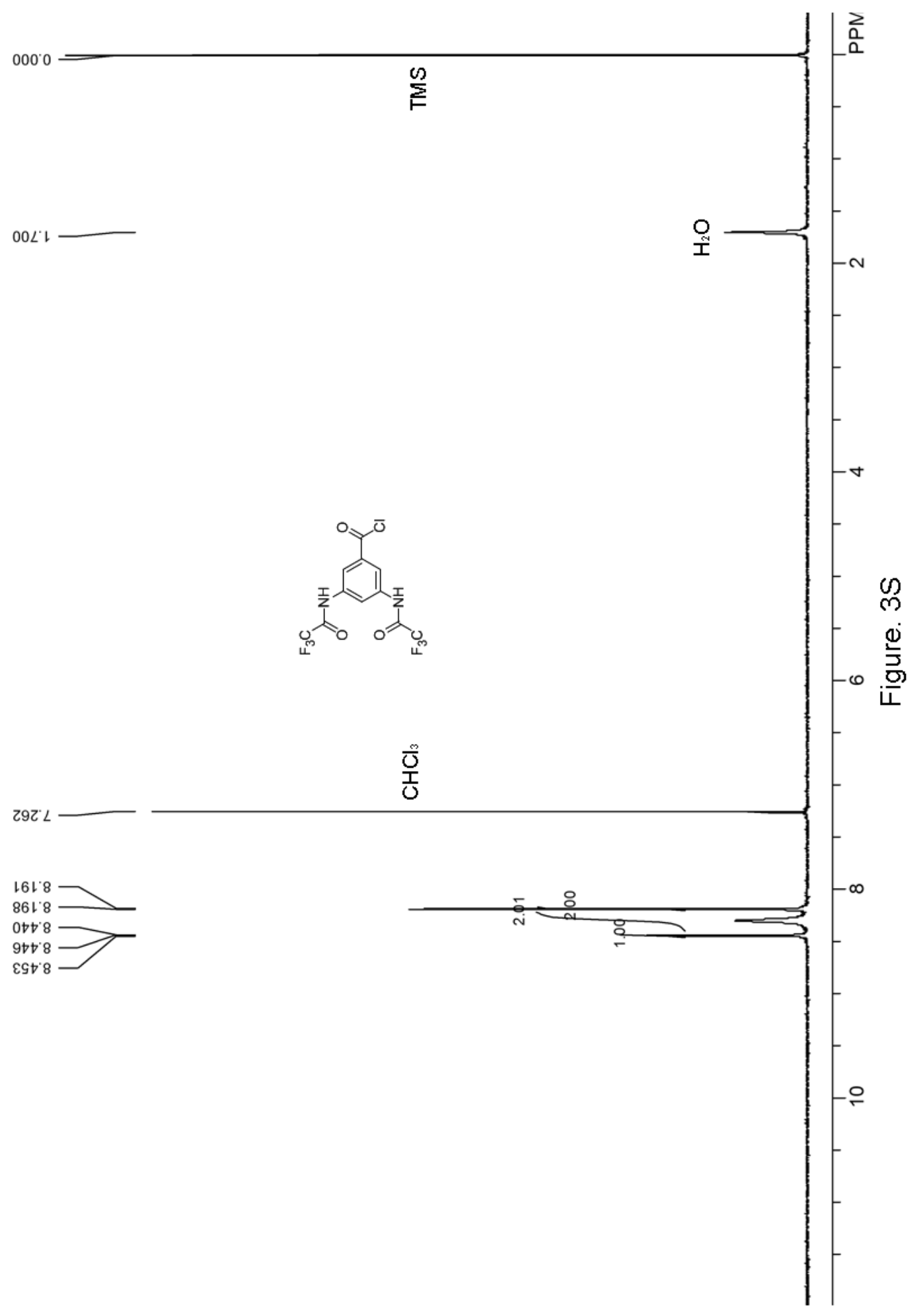


$0000-1$

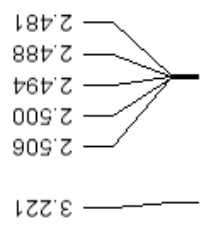

$8 \varepsilon 8^{\prime} \bullet-\longrightarrow$

$879^{\circ} \mathrm{L}-\longrightarrow$
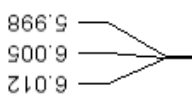

$\varepsilon เ \varepsilon^{\circ}$

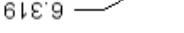

$\angle S L 6$

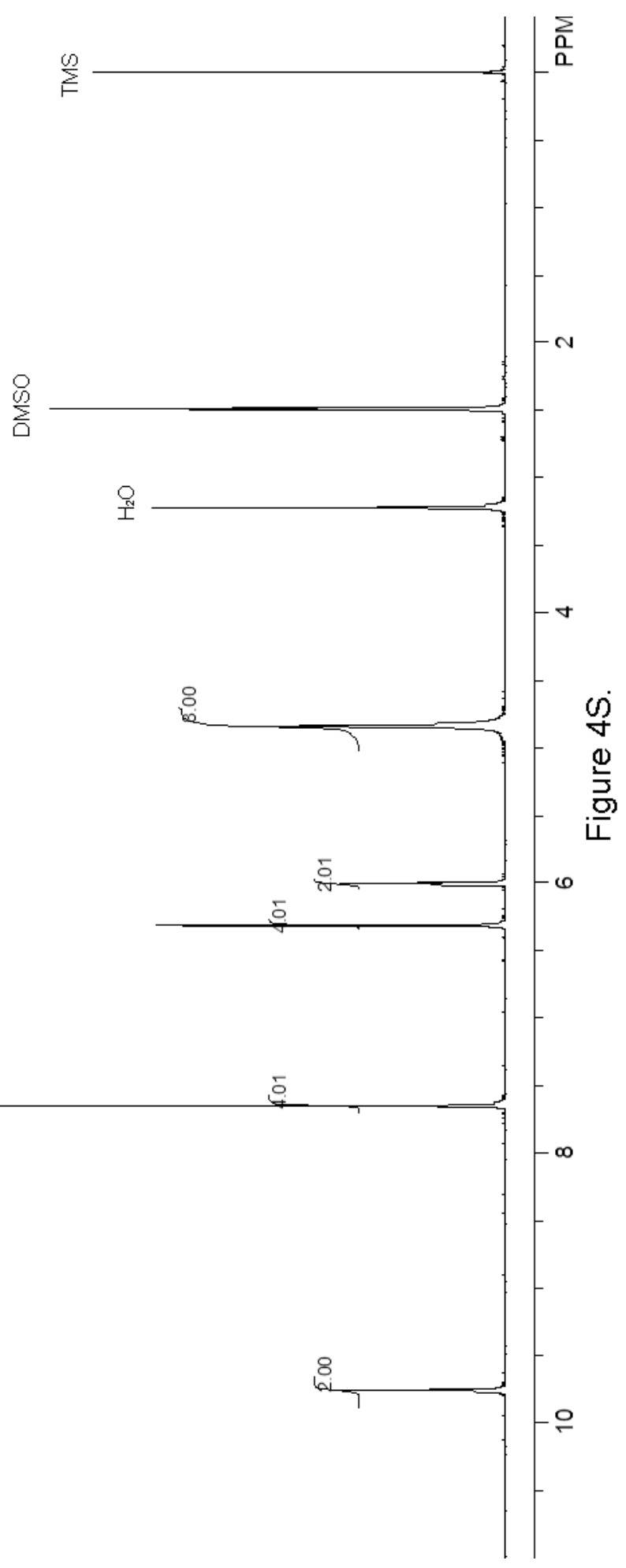



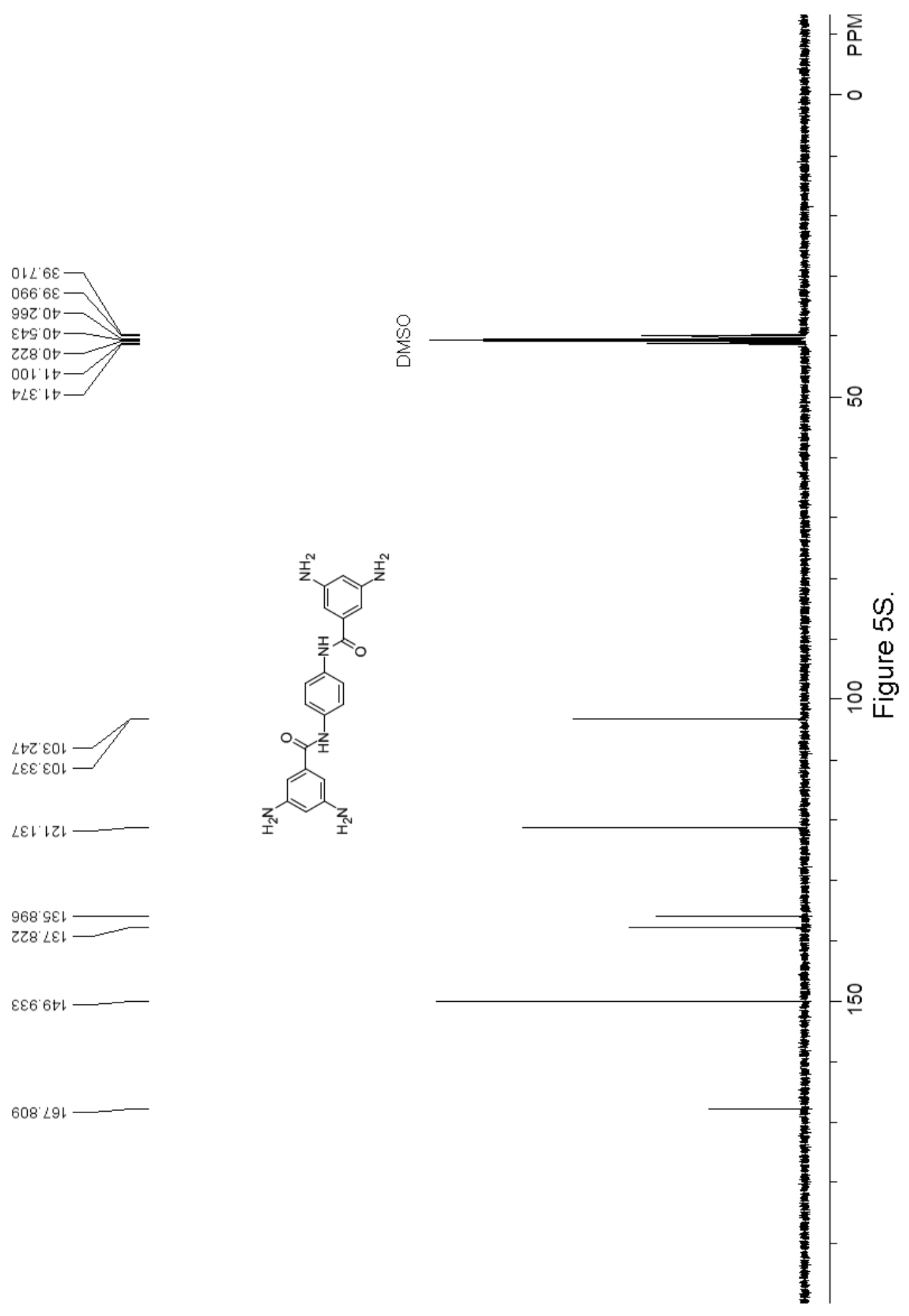

$9688^{\circ} \subseteq \varepsilon$

乙Z8 $\angle \varepsilon 1$

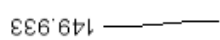

$608 \angle 91-\longrightarrow$

ก 


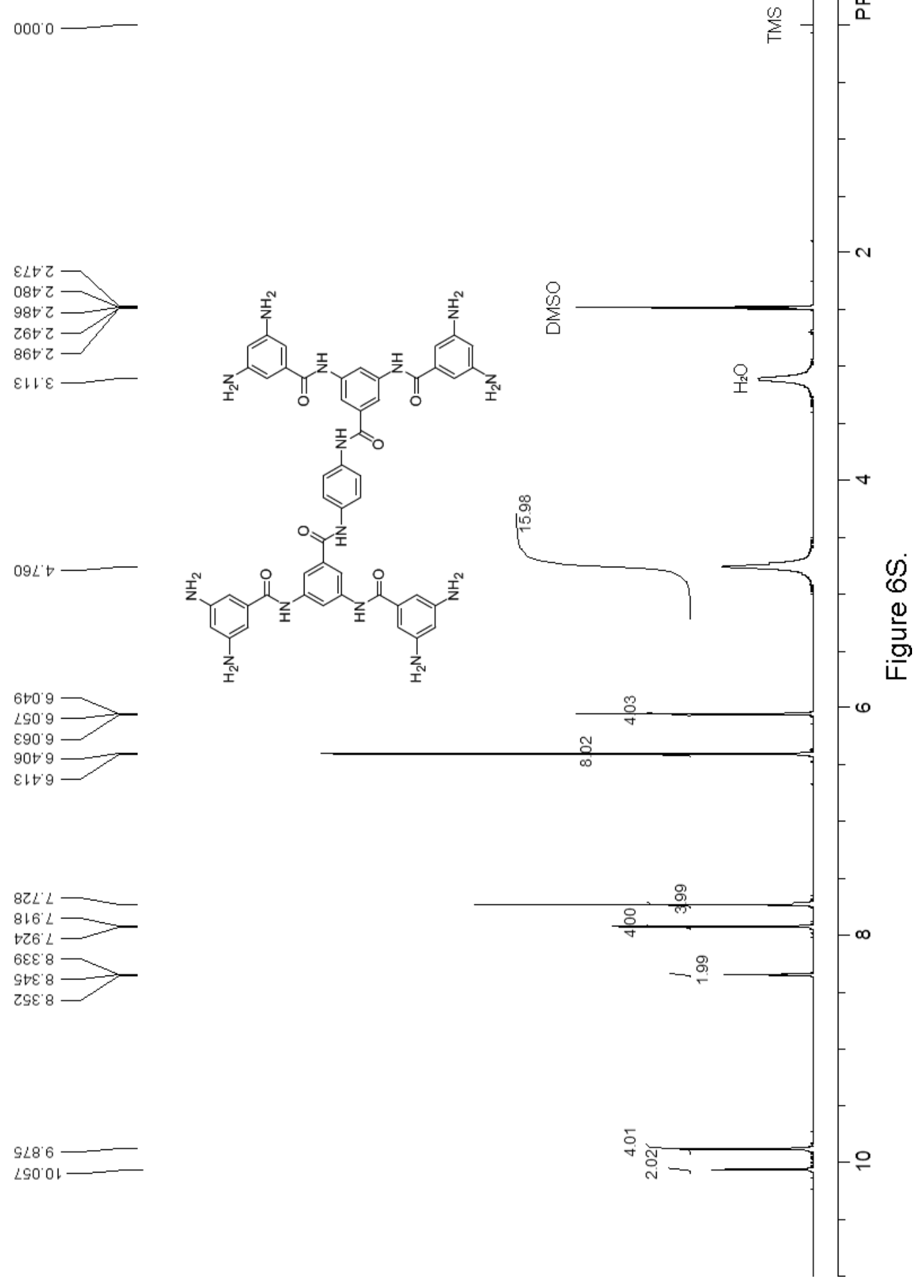




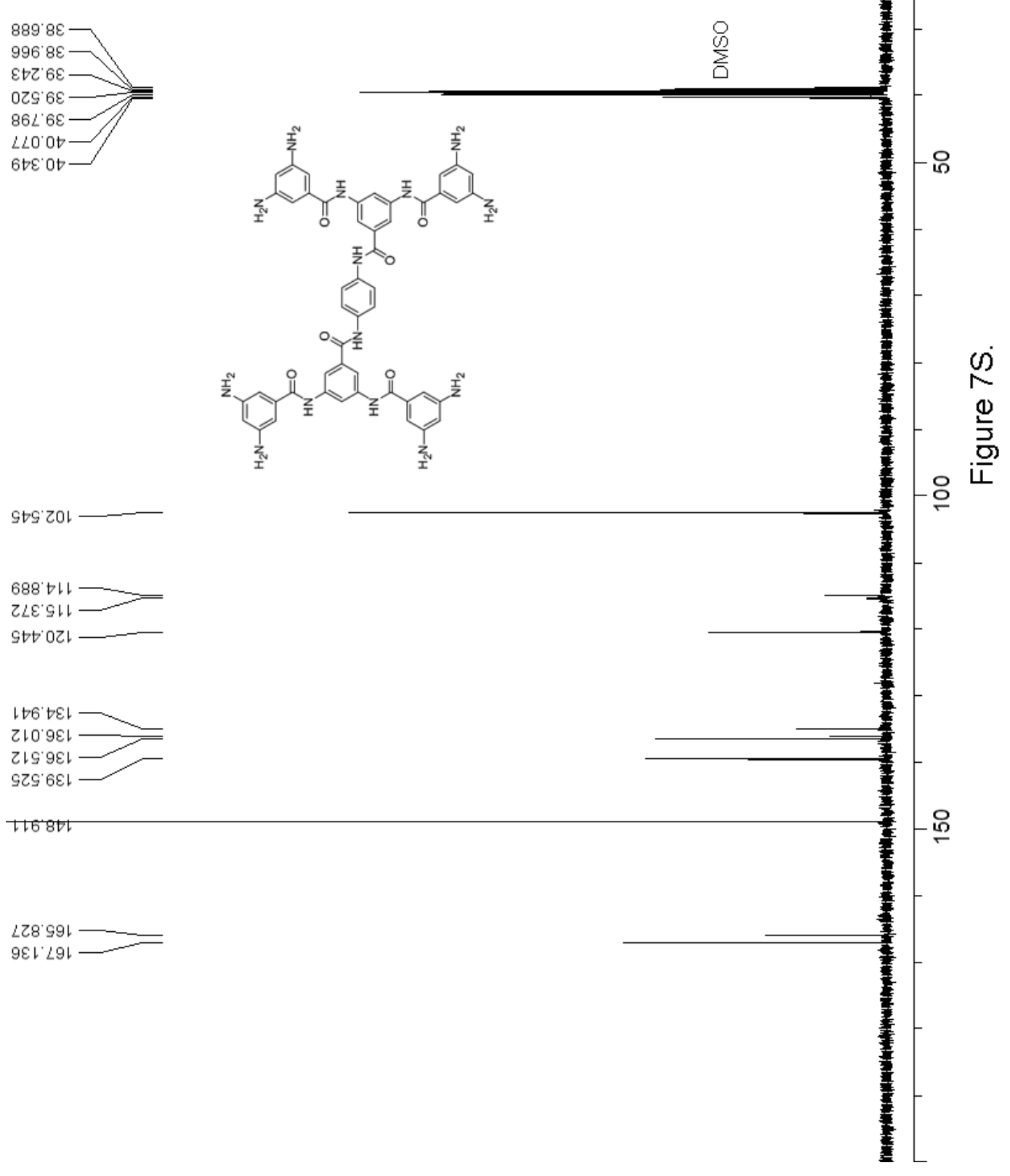




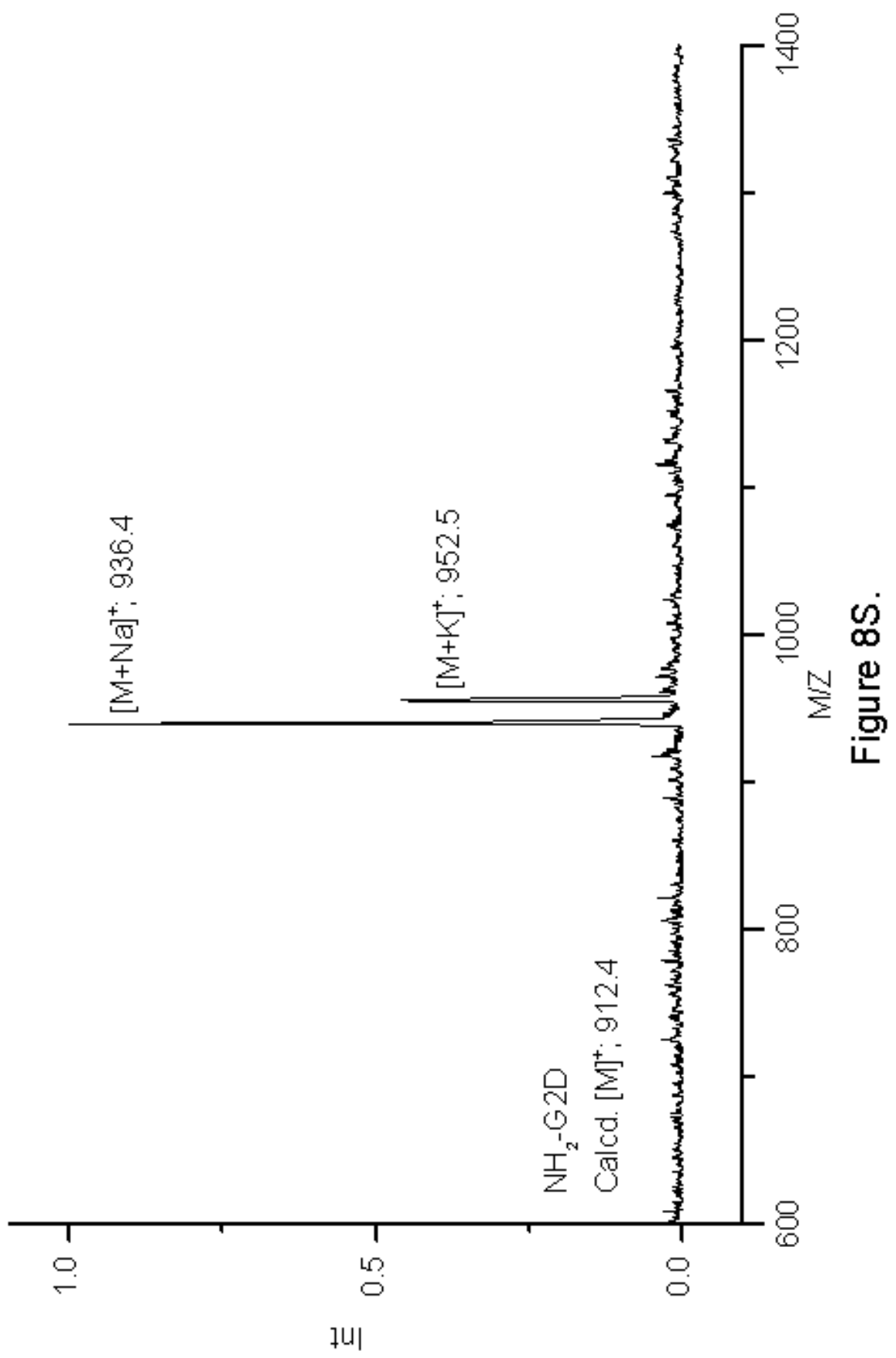


$0000 \div$
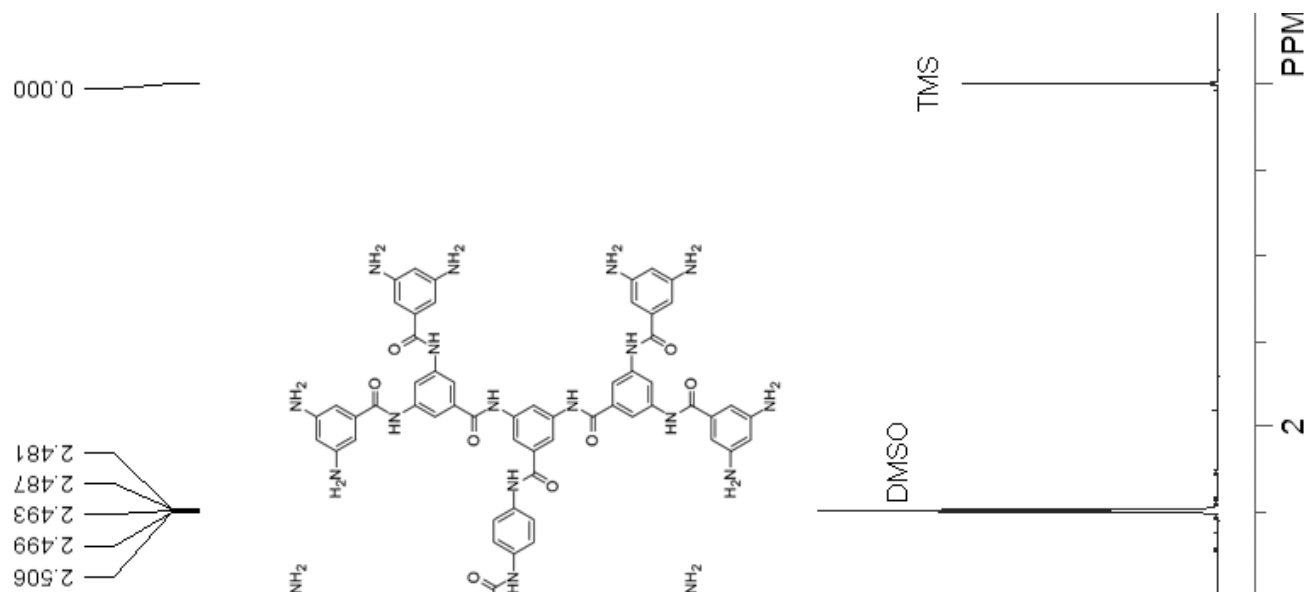

$s+Z^{\prime} \varepsilon$

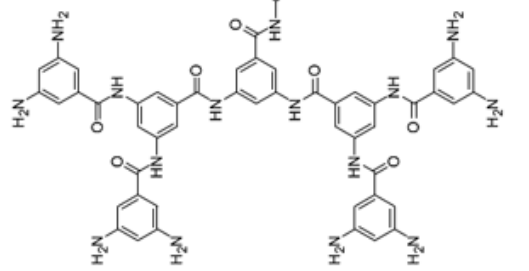

i)

$Z \angle 8 \circ \longrightarrow$

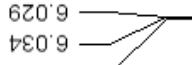

乙†0 9

$188^{\prime} 9$

$88 \varepsilon^{\prime} 9$

$0 \angle L L$

$286^{\circ} \angle$

$886 \angle \longrightarrow$

020

920.8

$->$

$69 \varepsilon^{\prime} 8$

$S L E$ ' $-\longrightarrow$

$86 t^{\circ} 8$

$\angle D O O L-C$

$96201-2$

$\angle 9 D^{\circ} \mathrm{Ol} \longrightarrow \longrightarrow$

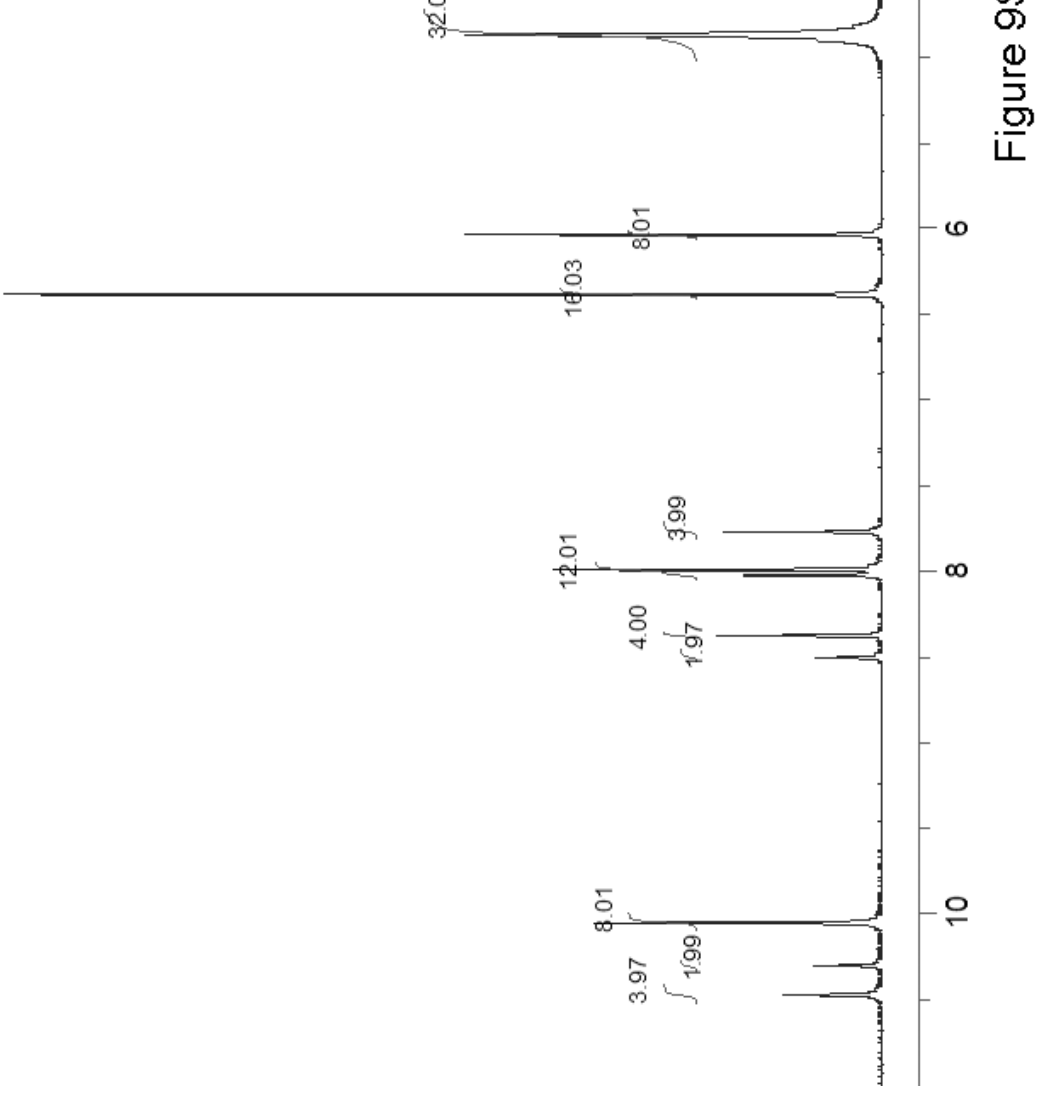




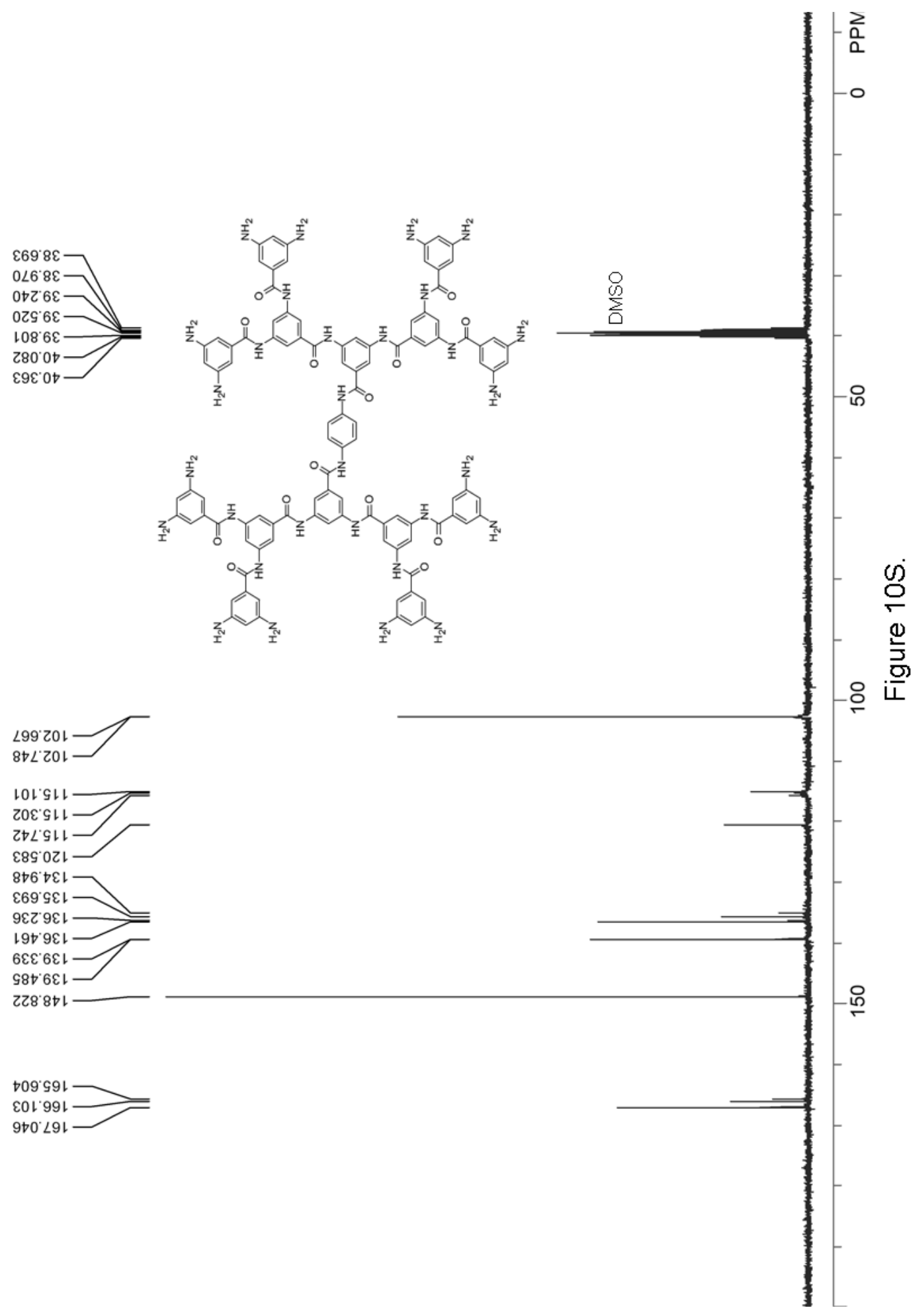




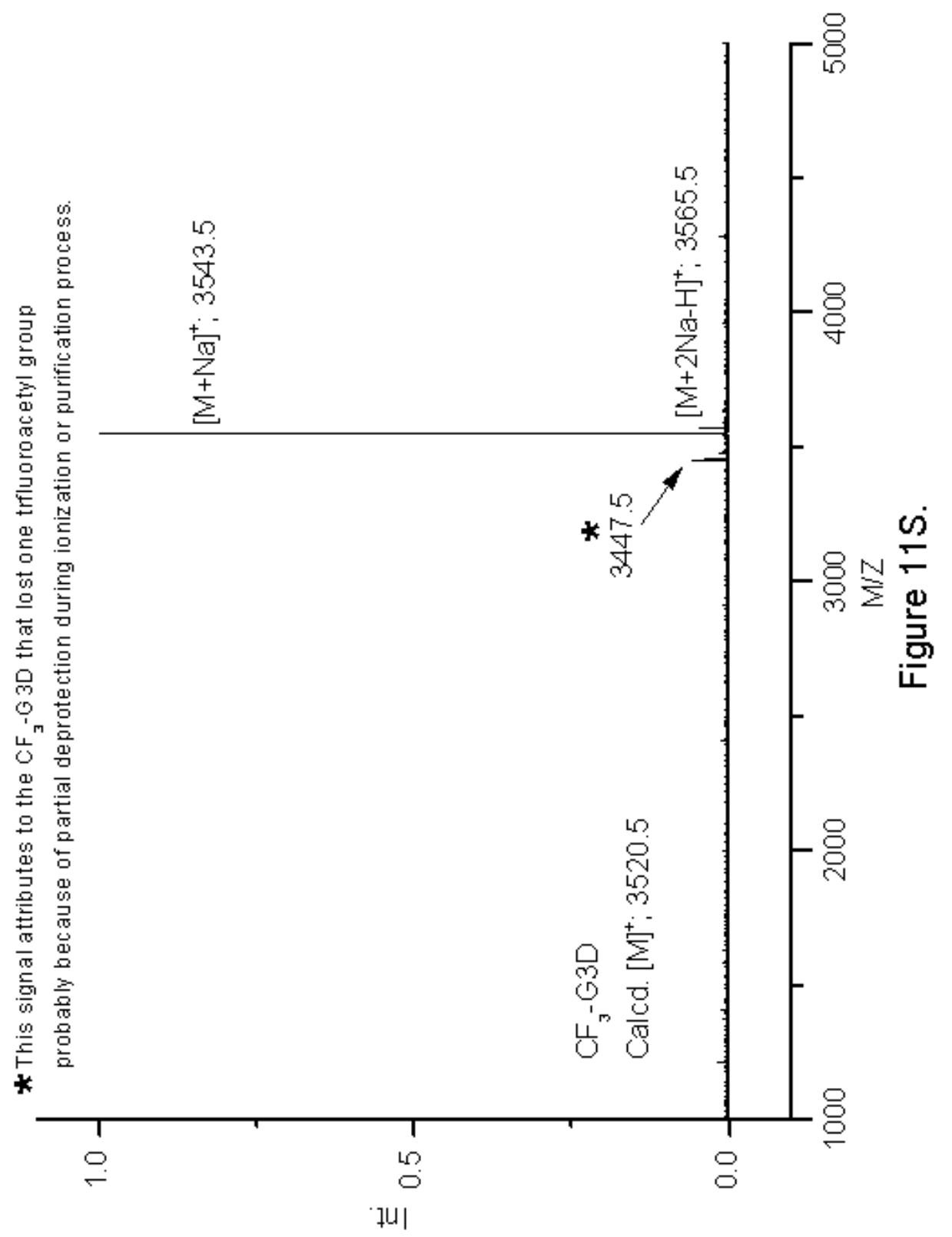




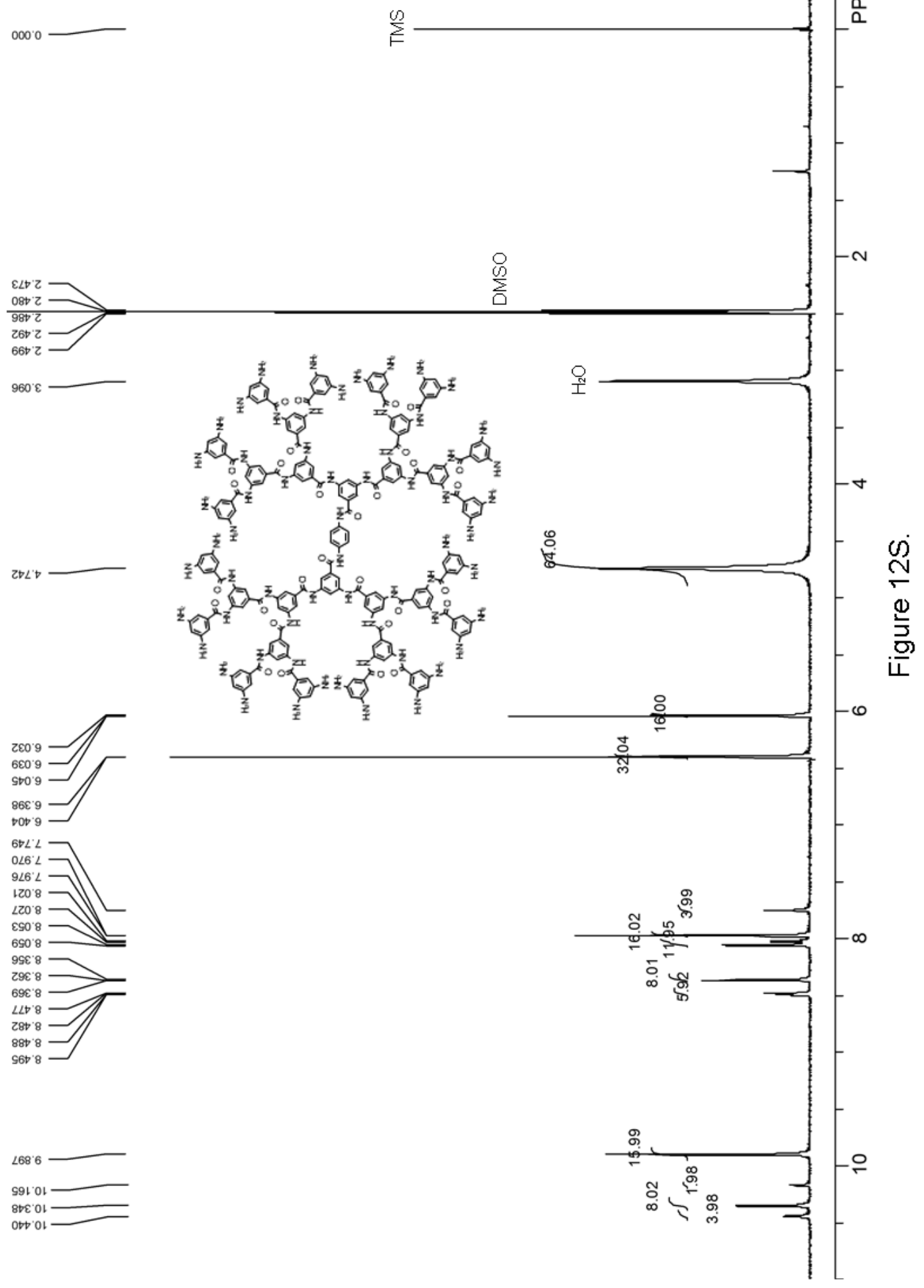




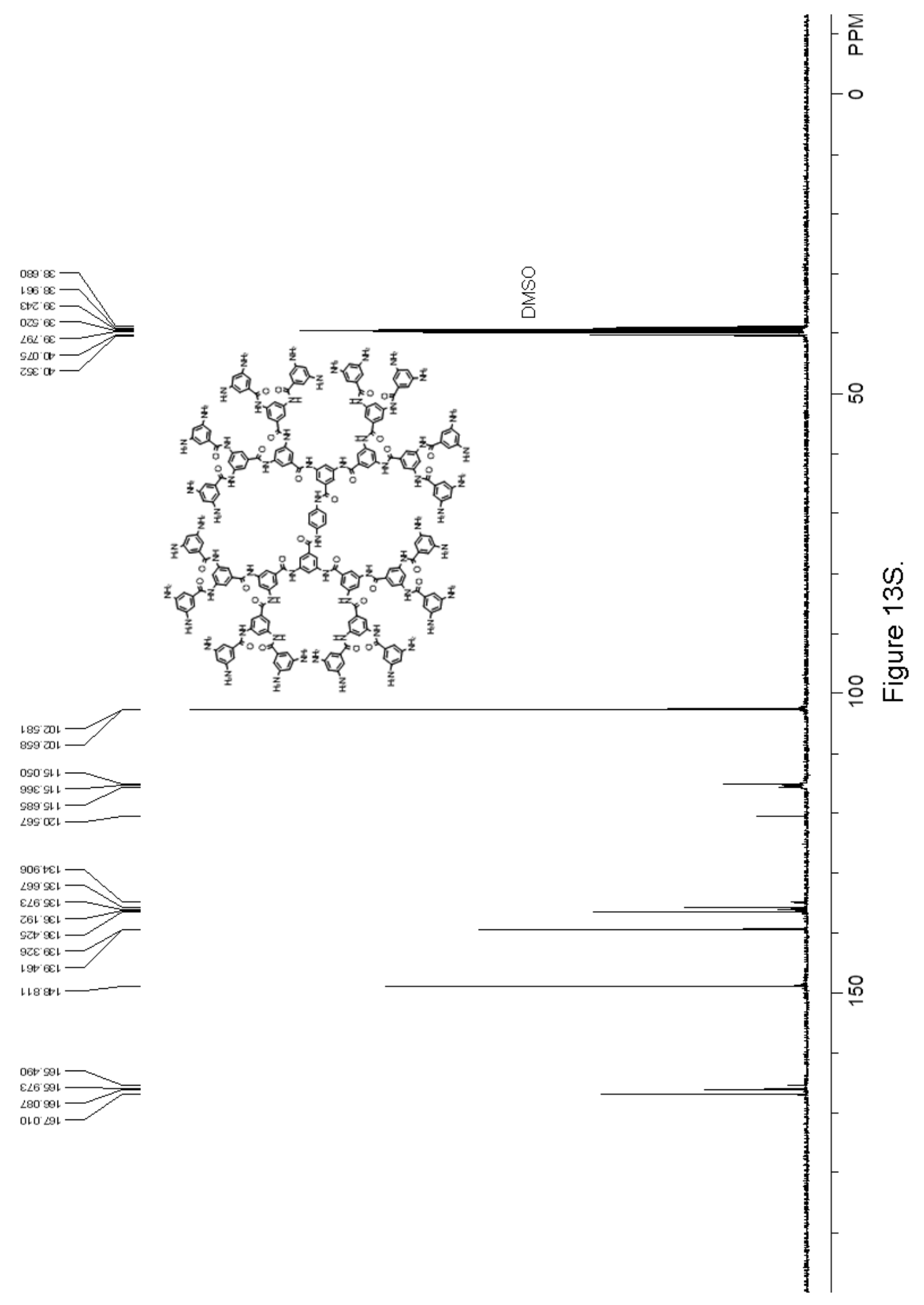




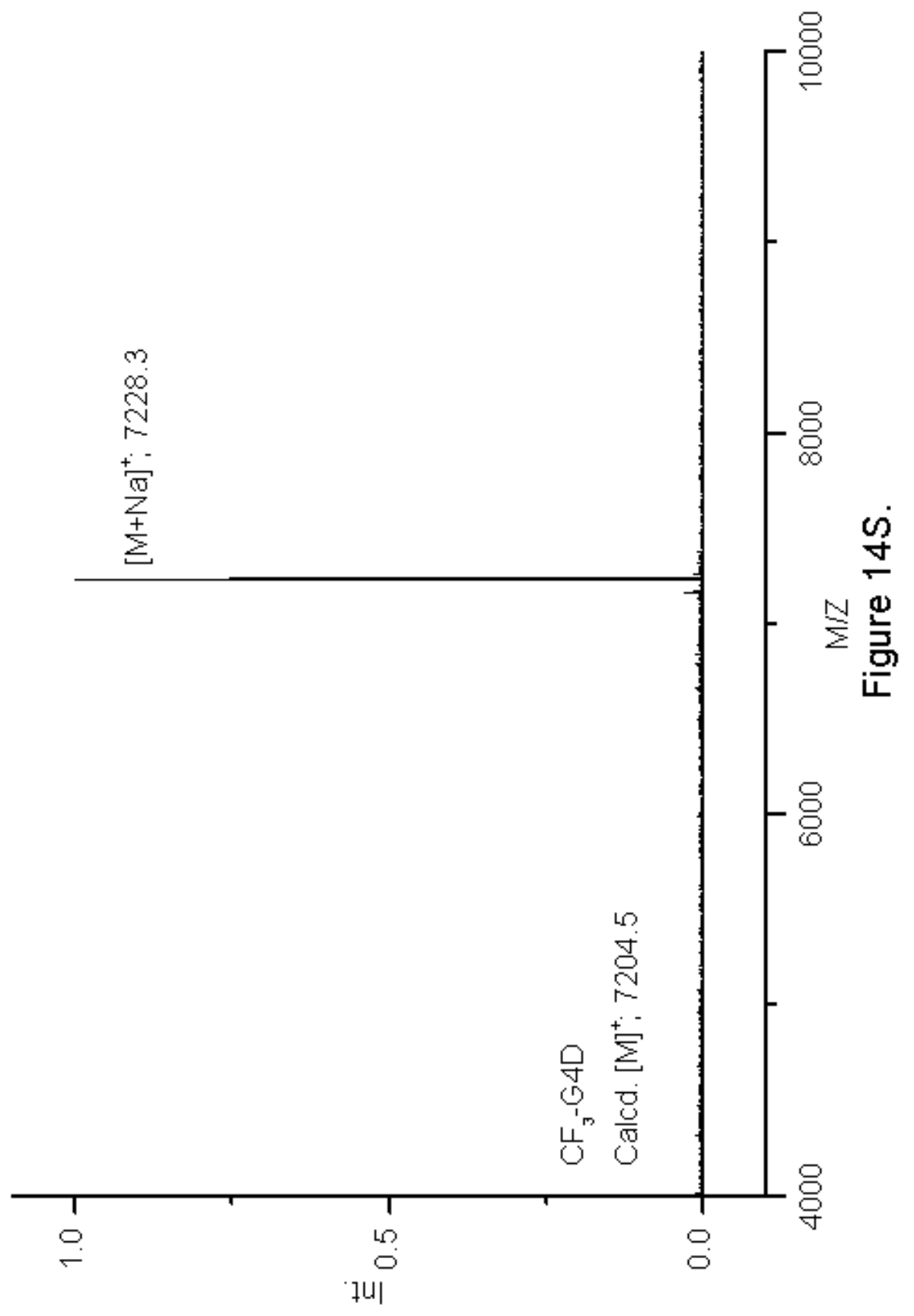



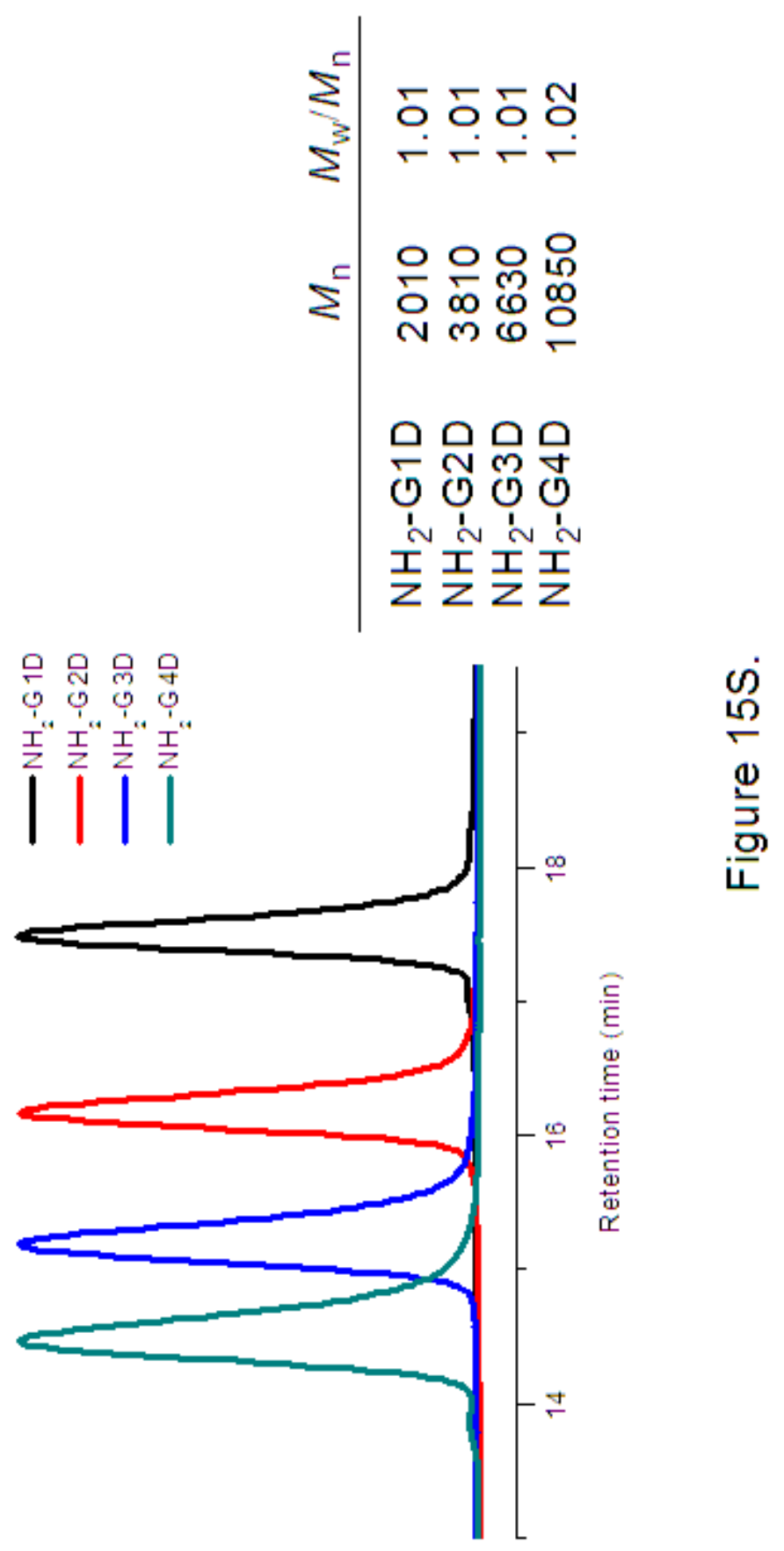